\title{
A New Current-Mode Universal Filter Using CCII and OTAs
}

\author{
Chih-Lung Lin , Ro-Min Weng, Maw-Huei Lee and Te-Son Kuo \\ Rm.537 Dept. of Electrical Engineering National Taiwan University \\ No.1 Sec 4, Roosevelt Rd., Taipei, Taiwan, R.O.C. \\ Tel: 886-2-23635251 Ext. 537 Fax: 886-2-23638247 \\ Email : cllin@cc.ee.ntu.edu.tw
}

\begin{abstract}
A new novel current-mode biquad filter using one multiple output second generation current conveyor (CCII), two operational transconductance amplifiers (OTAs), and two grounded capacitors is presented. The filter has three inputs and one output and can realise five different biquad transfer functions simultaneously without any changes in the circuit topology. This circuit enables the circuit characteristics to be electronically tuned. Grounded capacitors are suitable for integrated circuit implementation. An example is given, together with simulated results by PSPICE.
\end{abstract}

\section{Introduction}

The designs of current-mode circuits employing active devices such as CCII and OTA have been reported in the literature [1-2], The CCIIs and OTAs have been found useful in many applications, such as the synthesis of various active filter transfer functions. The $\mathrm{CCII}$ have been demonstrated to provide high performance than the conventional op-amps. Whereas, OTAs provide a highly linear electronic tunability and a wide tunable range of its transconductance gain. The circuit configuration consisting of OTAs, CCII, and grounded capacitors without resistors is considered to be more suitable for monolithic integration. Several designs with three input and one output current-mode universal filter have been discussed [3-4]. This Letter proposes a new universal filter circuit which need fewer active and passive elements than [3-4] to implement all the basic secondorder filter functions.

\section{Circuit Description}

The circuit of the proposed filter is shown Fig. 1. Using standard notation, the three output CCII \pm can be characterised by $\mathrm{i}^{z}= \pm \mathrm{i}^{\mathrm{x}}, \mathrm{i}^{\mathrm{x}}=0$, and $\mathrm{v}^{\mathrm{X}}=\mathrm{v}^{\mathrm{y}}[$ [5-6]. An OTA is a differential voltage controlled current source, and its circuit diagram is shown in Fig. 2. The

transconductance $g_{m}$ represents the ratio of the output current to the input voltage and it is adjustable within the range of several decades by a supplied bias current $I_{b}$.i.e. $g_{m}=I_{O U T} / V_{d}=I_{b} / 2 V_{T}$. Routine circuit analysis yields the current transfer functions:

$$
I_{\text {OUT }}=\frac{I_{1}\left(S^{2} C_{1} C_{2}\right)-I_{2}\left(S C_{2} g_{3}\right)+I_{3}\left(g_{3} g_{4}\right)}{S^{2} C_{1} C_{2}+S C_{2} g_{3}+g_{3} g_{4}}
$$

Five types of biquadratic filters are realised with the following specialization :

(i) highpass: $I_{2}=I_{3}=0$, input signal is $I_{1}$;

(ii) lowpass : $I_{1}=I_{2}=0$, input signal is $I_{3}$;

(iii) bandpass: $I_{1}=I_{3}=0$, input signal is $I_{2}$;

(iv) notch: $I_{2}=0$ and $I_{1}=I_{3}=$ input signal;

(v) allpass: $I_{1}=I_{2}=I_{3}=$ input signal;

The angular frequency $\omega_{0}$ and quality factor $Q_{0}$ are given by

$$
\begin{aligned}
\omega_{0} & =\left(\frac{g_{3} g_{4}}{C_{1} C_{2}}\right)^{1 / 2}=\frac{1}{2 V_{T}}\left(\frac{I_{b_{3}} I_{b_{4}}}{C_{1} C_{2}}\right)^{1 / 2} \\
\mathrm{Q}^{0} & =\left(\frac{C_{1} g_{4}}{C_{2} g_{3}}\right)^{1 / 2}
\end{aligned}
$$

Thus, the quality factor $Q_{0}$ can be controlled by the ratio of $\mathrm{C}_{1} / \mathrm{C}_{2}$ or by the transconductance gain ratio $g_{4} / g_{3}$ (i.e. adjusting the bias current $I_{b_{4}}$ and $I_{b_{3}}$ ).

From eqns. 2 and 3 it is easy to show that the passive sensitivities of the parameters $\omega_{0}$ and $Q_{0}$ are

$$
\begin{gathered}
\mathrm{S}_{C_{1}}^{\omega_{0}}=\mathrm{S}_{C_{2}}^{\omega_{0}}=-\mathrm{S}_{g_{3}}^{\omega_{0}}=-\mathrm{S}_{g_{4}}^{\omega_{0}}=-\frac{1}{2} \\
\mathrm{~S}_{C_{1}}^{Q_{0}}=-\mathrm{S}_{C_{2}}^{Q_{0}}=-\mathrm{S}{\underset{g}{3}}_{g_{0}}^{Q_{3}}=\mathrm{S} \frac{Q_{0}}{g_{4}}=\frac{1}{2}
\end{gathered}
$$

\section{Experimental Results}

To evaluate the performance of the circuits shown in Fig.1, consider the biquad characteristics with the chosen values for passive components: $\mathrm{C}_{1}=\mathrm{C}_{2}=318 \mathrm{pF}$, and $g_{3}=g_{4}=0.2 \mathrm{~ms}$. This choice leads to $\mathrm{f}_{0}$ $=\omega_{0} / 2 \pi=100 \mathrm{kHz}$, and $Q_{0}=1$. In PSPICE simulation , A CA3080 OTA macro model [7] has been used. The current-mode frequency responses of the highpass, bandpass, and lowpass filter are showing in Fig. 3. The simulation results confirm the theoretical analysis.

\section{Conclusions}

In summary, the proposed new current-mode universal biquad filter with three inputs and one output 
using only one three-ouput CCII, two OTAs, two grounded capacitors is presented. The circuit provide the following advantages:

(i) realisation of highpass, bandpass, lowpass, notch, allpass filtering from the same configuration.

(ii) grounded capacitors without resistors are suitable for integrated circuit implementation.

(iii) no requirement for matching components.

(iv) low sensitivities and fewer components.

\section{References}

[1] J. Ramirez-Angulo, and E. Sanchez-Sinencio, "High frequency compensated current-mode ladder filters using multiple output OTAs", IEEE Transaction on Circuits and Systems, 41, pp. 581-586 1994.

[2]T. Tsukutani, M. Ishiad, S. Tsuiki, and Y. Fukui, "Current-mode biquad without external passive elements", Electron. Lett., 32, pp. 197-198, 1996.

[3]C. M. Chang, C. C. Chien, and H. Y. Wang, "Universal active current filter with three inputs using current-conveyor II", International Journal of Electronics, 76(1), pp. 87-89, 1994.

[4]J. W. Horng, M. H. Lee, and C. L. Hou, "Universal active filter using four OTAs and one CCII", International Journal of Electronics, 78, pp. 903-906, 1995.

[5]T. A. Muhammad, and A. A. Muhammad, "Currentmode universal filters using-unity gain cells", Electron. Lett., 32, pp. 1077-1078, 1996.

[6]J. Wu, E. Ei-Masry, "Current-mode ladder filters using multiple output current conveyors", IEE Proc.Circuit Devices Syst., 143, pp. 218-222, 1996.

[7]J. Wu, "Current-mode high-order OTA-C filters", International Journal of Electronics, 76, pp. 1115$1120,1994$.

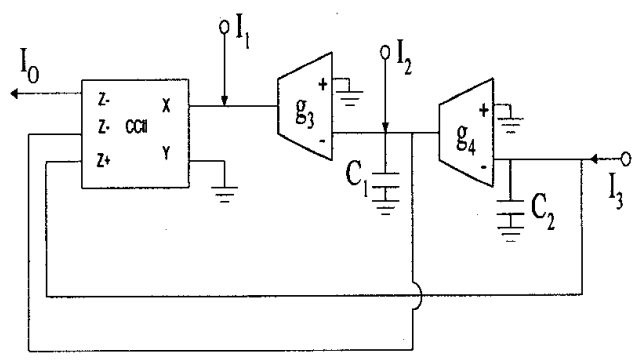

Fig. 1. Proposed universal biquad filter

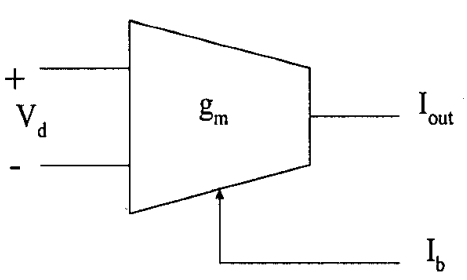

Fig. 2. Symbol of OTA

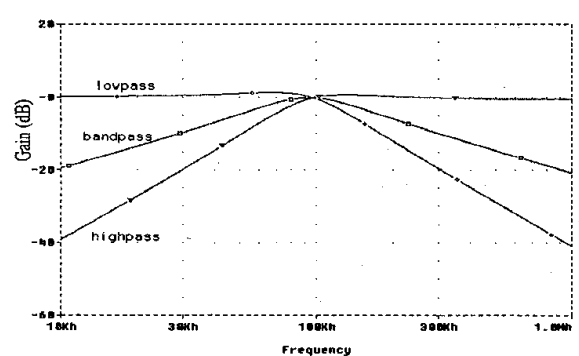

Fig. 3 Simulated results of lowpass, bandpass, and highpass responses 